\title{
Embryologic and Fetal Development of the Human Eyelid
}

\author{
Hatem A. Tawfik, M.D. *, Mohamed H. Abdulhafez, F.R.C.S. *, Yousef A. Fouad, M.B.B.S.ł, \\ and Jonathan J. Dutton, M.D., Ph.D., F.A.C.S.† \\ *Department of Oculoplastic Surgery, Watany Eye Hospital, Cairo, Egypt; †Department of Ophthalmology, University \\ of North Carolina School of Medicine, Chapel Hill, North Carolina, U.S.A.; and $\ddagger$ Department of Ophthalmology, Ain \\ Shams University, Cairo, Egypt
}

Purpose: To review the recent data about eyelid morphogenesis, and outline a timeline for eyelid development from the very early stages during embryonic life till final maturation of the eyelid late in fetal life.

Methods: The authors extensively review major studies detailing human embryologic and fetal eyelid morphogenesis. These studies span almost a century and include some more recent cadaver studies. Numerous studies in the murine model have helped to better understand the molecular signals that govern eyelid embryogenesis. The authors summarize the current findings in molecular biology, and highlight the most significant studies in mice regarding the multiple and interacting signaling pathways involved in regulating normal eyelid morphogenesis.

Results: Eyelid morphogenesis involves a succession of subtle yet strictly regulated morphogenetic episodes of tissue folding, proliferation, contraction, and even migration, which may occur simultaneously or in succession.

Conclusions: Understanding the extraordinary process of building eyelid tissue in embryonic life, and deciphering its underlying signaling machinery has far reaching clinical implications beyond understanding the developmental abnormalities involving the eyelids, and may pave the way for achieving scar-reducing therapies in adult mammalian wounds, or control the spread of malignancies.

(Ophthal Plast Reconstr Surg 2016;32:407-414)

P renatal development in humans is divided into 2 periods: an embryonic period and a fetal period. The embryonic period begins with fertilization and ends 8 weeks later, and the fetal period extends from that point onwards until delivery. Since 1914, the staging of human embryos in the first 8 weeks has been divided into 23 Carnegie stages, with each stage representing an arbitrary point along the timeline of development. ${ }^{1}$ Studying eyelid embryogenesis is fraught with difficulties because major studies detailing human embryologic and fetal eyelid morphogenesis are limited, were mostly performed decades ago, and

\footnotetext{
Accepted for publication February 29, 2016.

The authors have no financial or conflicts of interest to disclose.

Address correspondence and reprint requests to Hatem A. Tawfik, M.D., 5 Ibn Elnafis St., Off Makram Ebeid St., Nasr City, Cairo, Egypt. E-mail: hatem35@gmail.com

Dr. R. Patrick Yeatts served as guest editor for this paper.

Copyright (C) 2016 The Authors. Published by Wolters Kluwer Health, Inc. on behalf of the American Society of Ophthalmic Plastic and Reconstructive Surgery, Inc. All rights reserved. This is an open-access article distributed under the terms of the Creative Commons Attribution-Non CommercialNo Derivatives License 4.0 (CCBY-NC-ND), where it is permissible to download and share the work provided it is properly cited. The work cannot be changed in any way or used commercially.

DOI: 10.1097/IOP.0000000000000702
}

were usually focused on development during the embryonic rather than the fetal period. But some newer more comprehensive cadaver studies are now available, and dozens of recent articles document the molecular basis of eyelid development. Reviewing the literature spanning almost a century is confusing because some authors only referred to Carnegie stages, others liberally interchanged gestational age with postfertilization age, and some researchers used the crown-rump length measurements to determine the embryonic stage and fetal age, which may not be a very reliable measure after the $84 \mathrm{~mm}$ cutoff point set by some obstetricians. ${ }^{2}$ Other authors discussed the timeline of eyelid development in terms of weeks or months without specifying how the age was estimated. To accommodate old and new studies, to avoid confusion with nomenclature alien to Ophthalmologists, and for the sake of standardization, throughout the current manuscript the age mentioned in weeks is the postfertilization age with no reference to gestational age. All the measurements in $\mathrm{mm}$ refer to the crown-rump length, and any reference to Carnegie staging is disregarded. For the sake of brevity, the authors limit the current discussion to eyelid morphogenesis alone ignoring ocular embryogenesis or congenital eyelid defects. These topics have already been studied extensively, and pertinent literature can be sought elsewhere.

To understand eyelid development, a basic appreciation of embryonal development, particularly integumentary system embryogenesis, is important. In early embryonic life, the 3 primary germ layers are the ectoderm on the outside, the endoderm on the inside, and the mesoderm in between. Generally speaking, the mesoderm later gives rise to a loosely woven tissue called the mesenchyme among other components in the developing embryo. The surface ectoderm is initially a single cell layer thick. It later proliferates forming a new outer transient layer of simple squamous epithelium called the periderm cells, and an underlying proliferating layer called the basal layer, which is separated from the underlying dermis by a basement membrane. ${ }^{3}$ Later on, around the 11th week of fetal life, a third layer is produced deep to the periderm layer called the intermediate cell layer. ${ }^{3}$ Cells in this layer are called keratinocytes and are the forerunners of the outer layers of the mature epidermis. ${ }^{3}$ The basal layer, or stratum germinativum, constitutes the stem cell layer that will renew the epidermis throughout life. On the other hand, the most superficial layer, or the periderm cell layer, is of transient nature and is gradually shed into the amniotic fluid until it completely disappears by the 21 st week. ${ }^{3,4}$

Eyelid morphogenesis is a dynamic process involving complex interactions between the epidermis and dermis. ${ }^{5,6} \mathrm{~A}$ plethora of signaling molecules and pathways appear to coordinate 2 major tissue interactions during early eyelid development: intraepithelial and epithelial-mesenchymal interactions. Both types of interactions are integral for normal eyelid formation.? Overall, the eyelids develop from both secondary mesenchyme (mesoderm invaded by cranial neural crest cells) and surface 


\begin{tabular}{|c|c|}
\hline Mesoderm & Neural crest cells \\
\hline Fibers of striated muscles & Tarsal plate \\
\hline \multirow[t]{6}{*}{ Endothelial lining of eyelid blood vessels } & Levator aponeurosis, and Tarsal muscle of Müller \\
\hline & Connective tissue components of the levator and orbicularis muscles \\
\hline & Orbital septum \\
\hline & Eyelid melanocytes \\
\hline & Pericytes of eyelid blood vessels \\
\hline & Schwann cells and axons in eyelid nerves \\
\hline Surface ectoderm & Neuroectoderm \\
\hline Conjunctival and skin epithelium & None \\
\hline Hair follicles (mixed origin) & \\
\hline Zeis glands, glands of Moll & \\
\hline Meibomian glands (mixed origin) & \\
\hline Caruncle & \\
\hline
\end{tabular}

ectoderm. ${ }^{8-10}$ Generally, the surface ectoderm gives rise to the conjunctiva, skin epithelium, hair follicles, Zeis glands, glands of Moll, and meibomian glands, while the tarsal plate, levator muscle, orbicularis muscle, orbital septum, and tarsal muscle of Müller develop from the mesenchyme. ${ }^{11,12}$ Mesenchymal contribution to the developing eyelid primarily originates from the cranial neural crest cells, while the mesodermal contribution is only to blood vessels' endothelium and striated muscles in the eyelids and orbit (Table 1).$^{10}$ It is difficult, however, to strictly classify the origin of individual eyelid structures into an ectodermal or mesenchymal origin because some structures start off with the extension of an epithelial placode into an underlying mesenchymal component. ${ }^{16}$

Eyelid development has been divided into $3,{ }^{13} 4,{ }^{17}$ or even 5 distinct phases, ${ }^{14}$ namely eyelid formation, fusion, development, separation, and maturation of eyelid structures. The authors prefer to classify it in a more simple chronological fashion into embryonic and fetal stages because several events of those aforementioned phases can simultaneously coexist. Chronological data for eyelid development during the embryonic and fetal stages are summarized in Table 2.

\section{THE EYELID DURING THE EMBRYONIC STAGE}

Ocular and eyelid development occurs in a step-wise fashion, and any misstep will be followed by failure of subsequent steps. ${ }^{28}$ At week 5 (31-35 days, 5-7 mm stage), there is still no indication of eyelid fold development, but the lens pit starts to invaginate from the surface ectoderm. ${ }^{15}$ The first indication of the embryonic eyelid occurs around the beginning of week 6 (37-42 days, $8-11 \mathrm{~mm}$ stage) where small grooves or depressions develop in the surface ectoderm immediately above and below the developing eye, which rapidly deepen to form the eyelid folds. ${ }^{10,15}$ At this stage, the eyelid is a primitive structure consisting of surface periderm covering undifferentiated mesenchyme. By the end of week 6 (42-44 days, 11-14 mm stage), the upper eyelid fold, which develops from the frontonasal process, ${ }^{10}$ is barely visible and is less distinct than the lower eyelid fold which originates from the maxillary process, and initially develops first. ${ }^{10,15,18}$ Distinct upper and lower eyelid folds are well defined at week 7 (48-51 days, 16-18 mm stage) and at this stage, the upper eyelid starts to assume its dominant role compared with the lower eyelid. ${ }^{15}$ Conversely, Andersen et al. ${ }^{13}$ maintain that the upper eyelid is always more distinct than the lower starting as early as the $12 \mathrm{~mm}$ stage. Both folds are covered by epithelium on their anterior and posterior surfaces, with 2 layers of epithelium on the anterior surface and a single layer of epithelium on the posterior surface. ${ }^{14}$ Also during the 7th week (48-51 days, 16-18 $\mathrm{mm}$ stage), a solid cord of epithelial cells invaginates into the thickened mesenchyme in the medial 1/6th of the eyelid and bifurcates forming the precursors of puncta and canaliculi. . $^{14,18,29,30}$

At the beginning of week 8 (54-56 days, 23-28 mm), there is a gap between the upper and lower eyelid folds and the cornea is still visible. In an attempt to fill the gap, the flattened periderm cells undergo a morphogenetic change into rounded or cuboidal cells which proliferate and start to migrate centripetally toward each other from the rim of both eyelids. This begins the remarkable process of eyelid fusion just anterior to the horizontal equator of the globe (Fig. 1) ${ }^{7,10,31}$ The leading edge cells extend filopodia that scan and help make contact with the advancing edge of the opposing eyelid. ${ }^{32,33}$ When a connection is established between both sides, the periderm cells flatten again and form a continuous sheet, ultimately covering the cornea. This process of eyelid fusion involves 2 coordinated yet distinct processes: epithelial cell migration and proliferation of the epithelium at the migrating edge (Fig. 1). ${ }^{32-37}$ Crucial for eyelid closure is the accumulation and realignment of contractile elements (F-actin and myosin) at the leading edge cells, forming a "contractile cable" which pulls opposing epithelial sheets from both eyelids together in a pursestring fashion effecting a tight closure. , $^{735}$

Controversy exists over where eyelid fusion actually begins. Although some studies have persistently demonstrated that the upper and lower eyelids first meet at the lateral end of the palpebral fissure with fusion progressing medially in a zipperlike fashion, ${ }^{15,18-20}$ others have shown that fusion begins in the temporal and nasal canthi simultaneously and progresses toward the center of the eye in a purse-string fashion..$^{7,21,35}$ Further studies are required to demonstrate whether interspecies differences could explain the discrepancy.

Concomitant with this whole process, the reflected edge of the epithelium along the advancing eyelid margins also extends a less distinct covering of periderm cells along the conjunctival surface. ${ }^{14,34}$ According to Sevel, ${ }^{14}$ this single layer of epithelium (periderm cells) on the posterior eyelid surface will later form the tarsal conjunctiva that ultimately fuses with the bulbar conjunctiva.

Only the periderm and epidermal layers are involved in eyelid fusion (Fig. 1), while eyelid mesenchyme remains distinct and unfused in preparation for future separation, and the development of future adnexa of both eyelids. ${ }^{32}$ A thickened well-distinct basement membrane beneath the epithelial cap is postulated to prevent penetration of the mesenchyme into the fusing epithelial folds. ${ }^{13}$ However, this does not mean that the role of mesenchyme is entirely passive in the process of eyelid fusion. Some signaling molecules like fibroblast growth factor 10 (Fgf10), instrumental for coordinating mammalian epithelial eyelid fusion as well as corneal development, are expressed in 


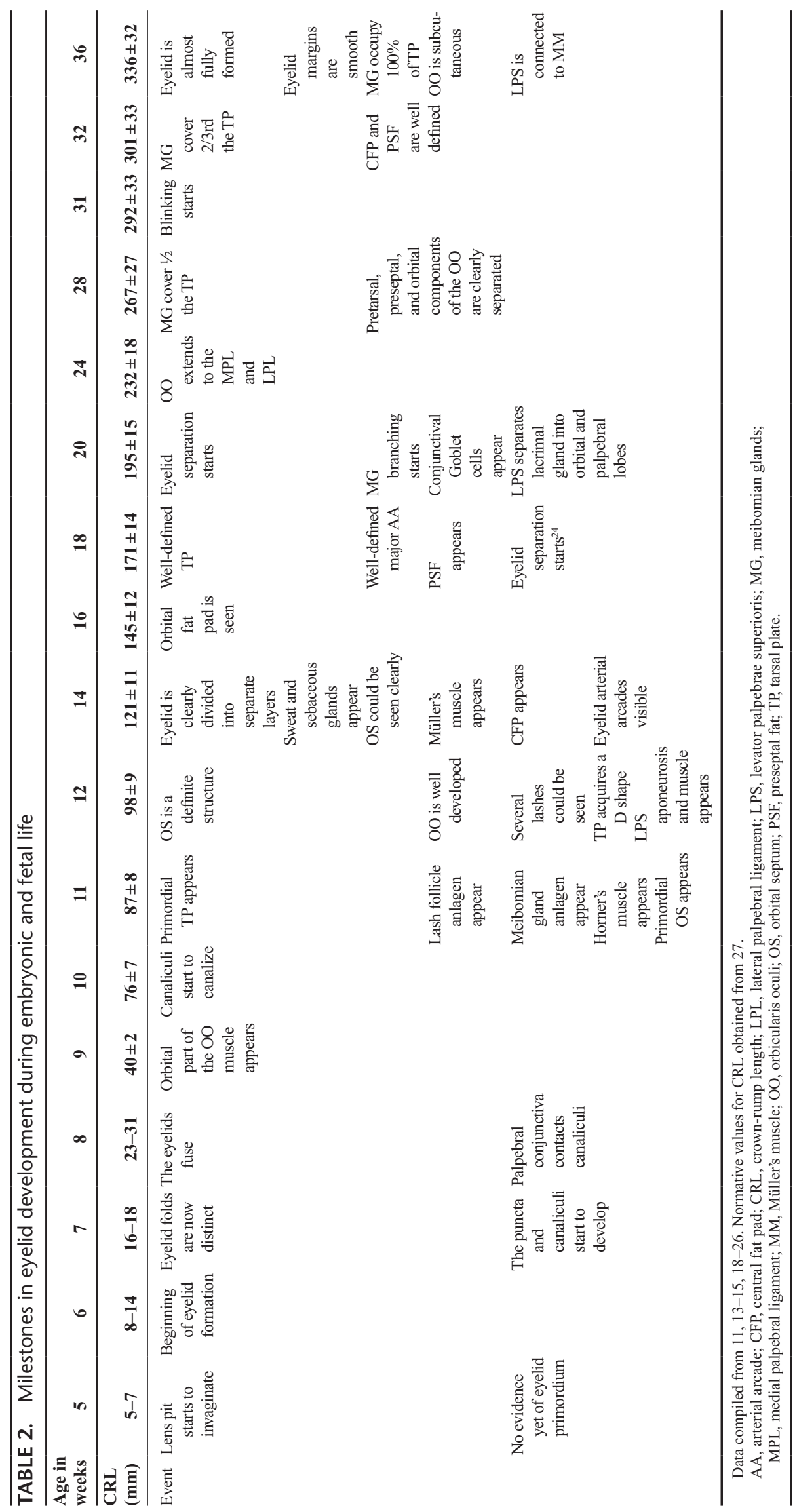




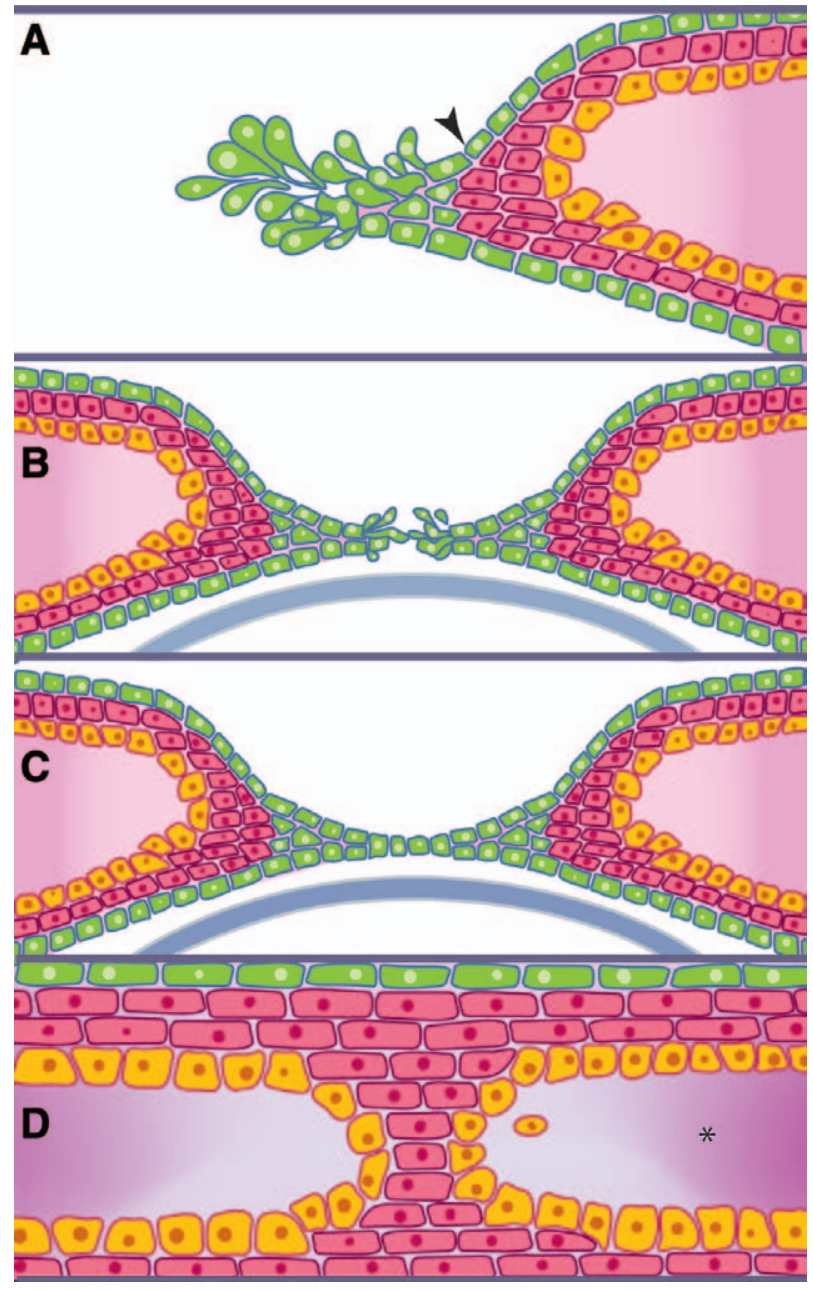

FIG. 1. The process of eyelid fusion. A, At around week 8, the flattened periderm cells (arrowhead) undergo a morphogenetic and proliferative change into rounded or cuboidal periderm cells. B, The leading edge of these proliferating cells helps make contact with the advancing edge of the opposing eyelid periderm cells until a connection is established. C, When a connection is established between both sides, these periderm cells flatten again and form a continuous sheet ultimately covering the cornea. D, Only the periderm and epidermal layers are involved in eyelid fusion while eyelid mesenchyme $\left(^{*}\right)$ remains distinct (Modified with permission from Yu et al. ${ }^{32}$ ).

the eyelids only in mesenchymal cells. ${ }^{7}$ By the end of week 8 (56-60 days, $27-31 \mathrm{~mm}$ ), the process of eyelid fusion is now complete, and this coincides with the conclusion of the embryonic stage and the beginning of the fetal stage..$^{15,18}$

\section{THE EYELID DURING THE FETAL STAGE}

The development of eyelid structures begins in the 9th week immediately following eyelid fusion. ${ }^{14}$ Mesenchymal cell condensations, coupled with an occasional ingrowth of surface epithelium into the underlying mesenchyme, contribute to the formation of some eyelid structures. ${ }^{14,18}$ The first to appear at week $9(40 \pm 2 \mathrm{~mm})$ is the orbital part of the orbicularis oculi muscle (Fig. 2A). Similar to other muscles innervated by the facial nerve, the orbicularis oculi muscle develops from the mesenchyme of the second pharyngeal arch and later migrates into the eyelids. ${ }^{9}$ The canaliculi start to canalize around week $10(76 \pm 7 \mathrm{~mm}) .{ }^{30}$
The primordial tarsal plate, followed by the eyelash follicle anlages, and the primordial orbital septum are likewise seen by the 11 th week $(87 \pm 8 \mathrm{~mm}) .{ }^{18,22}$ The first appearance of the meibomian gland anlages also occurs around week $11 .{ }^{13}$ After a series of signaling processes between the dermis and the overlying epidermis, the epithelial placodes of the meibomian anlagen dip or invaginate into the underlying mesenchymal condensation forming solid cords of cells that later form lateral outgrowths. These differentiate into connecting ductules and secretory holocrine sebaceous acini, while the central epithelial cylinder of the meibomian anlage forms a central canal that subsequently develops into the central duct. ${ }^{16,18,23}$ Meibomian gland development shows substantial resemblances to that of the eyelash follicles, and the meibomian gland is sometimes dubbed a "hair follicle without a hair shaft." ${ }^{23}$ Both anlages grow from the same ectodermal sheet, although the meibomian anlage grows deeper, and develops more slowly. ${ }^{13,23}$ This embryological mimicry also includes observation of keratohyalin granules in the luminal epithelium of the meibomian anlages, which may explain why hyperkeratinization is a typical disease of adult meibomian glands. ${ }^{23}$

By week $12(98 \pm 9 \mathrm{~mm})$, several eyelashes can be seen. The orbital part of the orbicularis oculi muscle is well developed. The levator muscle and aponeurosis, which develop from the same mesenchymal complex as the superior rectus and superior oblique muscles and tendons, starts to appear separately by a method of differential growth..$^{9,14,38}$ Throughout the embryonic and most of the fetal periods, the levator muscle shares a common epimysium with the superior rectus, which is initially quite dense and well demarcated. ${ }^{9,38}$ During the 12 th week, the levator aponeurosis is observed running downwards in close proximity to the primordium of the orbital septum. ${ }^{18}$ Müller's muscle, however, is not yet seen. ${ }^{14,18}$ The superior tarsal plate acquires a superiorly oriented D-shape and starts to merge with the levator aponeurosis, ${ }^{10,14}$ but the inferior tarsal plate remains sausage shaped. ${ }^{14}$ The marginal and peripheral arterial arcades are also visible anterior to the tarsal plate. ${ }^{18}$

During the 13th week $(109 \pm 10 \mathrm{~mm})$, the eyelash anlagen develop lateral outgrowths, ${ }^{13}$ which differentiate into the holocrine sebaceous glands of Zeis and modified sweat glands of Moll. ${ }^{13,23}$ By week $14(121 \pm 11 \mathrm{~mm})$, the eyelid is now clearly divided into separate layers, sweat and sebaceous glands appear, the orbital septum is well defined, and Müller's muscle makes its first appearance (Fig. 2B). The orbital fat, with its anterior extraconal component behind the orbital septum, is seen as a definite structure by week $16(145 \pm 12 \mathrm{~mm}) .{ }^{18}$ Also by week 16 , the canaliculi are clearly patent but the puncta remain closed until after the eyelids separate. ${ }^{21,30}$ Differential growth of the frontonasal and maxillary processes pushes the lower punctum in a more lateral position than the upper. ${ }^{30}$ The exact timing of development of the lacrimal caruncle is not entirely clear in the literature but generally is considered to develop "soon" after eyelid fusion. ${ }^{13,21}$ It is not formed from the conjunctiva but as an off-shoot from the lower eyelid. The developing lower canaliculus cuts the caruncle off from the remaining eyelid tissue. ${ }^{21}$

By week $18(171 \pm 14 \mathrm{~mm})$, the tarsal plates and the vascular arcades are well defined, and the meibomian gland anlages are now observed as a well-defined structure. ${ }^{18}$ By week 20 $(195 \pm 15 \mathrm{~mm})$, the lateral horn of the levator aponeurosis has expanded above the globe underneath the orbital roof and is now seen dividing the lacrimal gland into orbital and palpebral lobes. ${ }^{10,11,18}$ Meibomian gland branching is first observed during the 20th week (Fig. 2C). ${ }^{18}$ To the best of the authors' knowledge, the morphogenesis of lower eyelid retractors has not yet been reported in the literature.

There is some controversy regarding the events and timing of eyelid separation, but in general, separation starts 

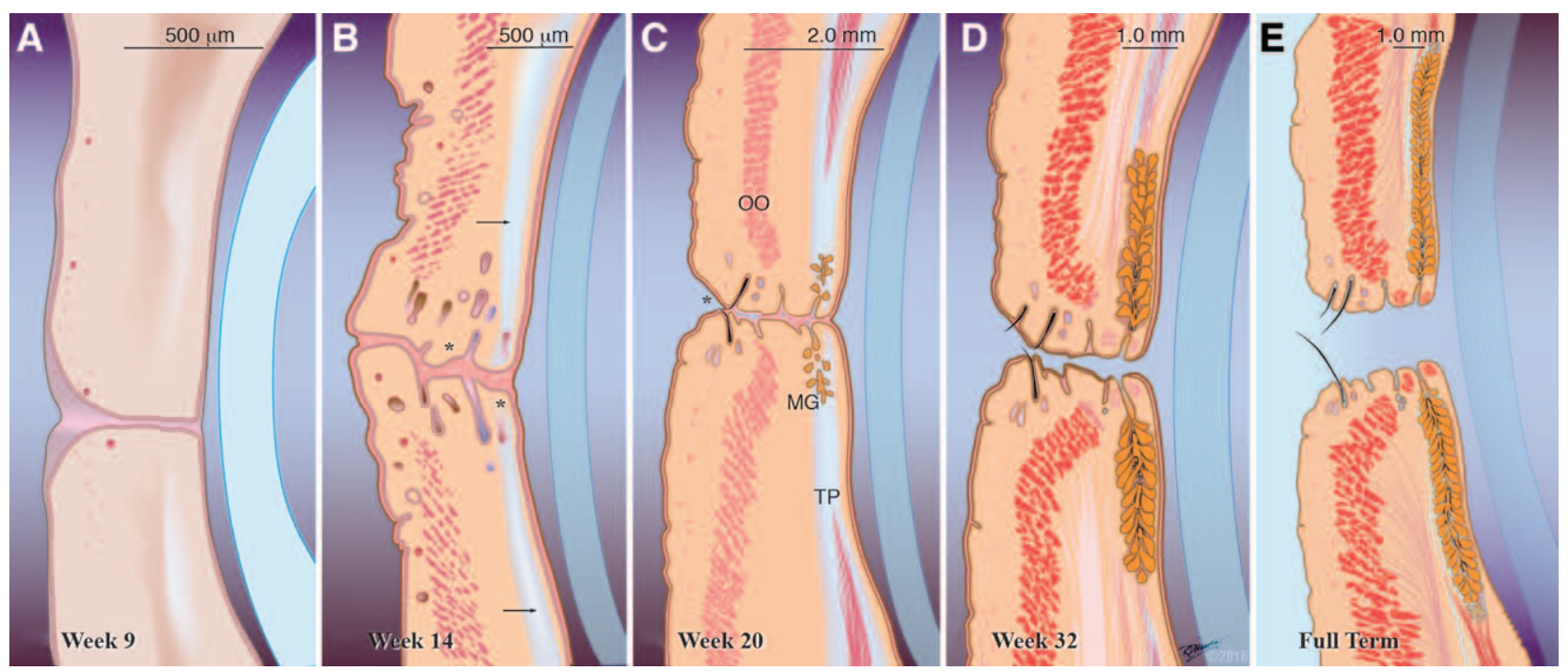

FIG. 2. Maturation of the eyelids during the fetal period. A, Week $9(40 \pm 2 \mathrm{~mm})$. The development of eyelid structures begins in the 9 th week immediately following eyelid fusion with mesenchymal cell condensations and an occasional ingrowth of surface epithelium into the underlying mesenchyme, which together contribute to the formation of some eyelid structures. The first to appear is the orbital part of the orbicularis oculi muscle. B, By week $14(121 \pm 11 \mathrm{~mm})$. The eyelid is now clearly divided into separate layers. Rudimentary eyelashes, sebaceous, and sweat glands $\left({ }^{*}\right)$ could be seen near the eyelid margin, and a primordial tarsal plate has formed (arrow). C, Week $20(195 \pm 15 \mathrm{~mm})$. Although the eyelids are still visibly fused, separation has already started anteriorly $\left(^{*}\right)$ and is visible in the middle at a microscopic level. Meibomian gland branching is first observed and the tarsal plate has lengthened significantly. The orbicularis oculi muscle looks more fully developed. Nearly mature eyelash follicles about to pierce the eyelid margin are also evident. D, Week $32(301 \pm 33 \mathrm{~mm})$. The eyelid has taken its nearly fully developed appearance. Meibomian glands increase in length and are present in two-thirds of the length of the tarsal plate. The eyelids are fully separated by now but the eyes are still visibly closed. E, Full term. Final appearance of the eyelids at birth, which is not dissimilar from the adult counterpart. OO, orbicularis oculi muscle; MG, Meibomian glands; TP, tarsal plate.

around the 20th week $(195 \pm 15 \mathrm{~mm}) \cdot{ }^{14,18}$ While Doxanas and Anderson' ${ }^{22}$ frequently quoted figure marks the beginning of eyelid separation at 24 weeks $(232 \pm 18 \mathrm{~mm})$, Byun et al. ${ }^{18}$ have observed that eyelid separation starts at a slightly earlier date (week 20,195 $\pm 15 \mathrm{~mm}$ ). In his histologic study, Sevel ${ }^{14}$ demonstrated that eyelid separation starts earlier around the 18th week $(171 \pm 14 \mathrm{~mm})$, while Andersen et al. ${ }^{13}$ reported an even earlier date (week 16, 145 $\pm 12 \mathrm{~mm}$ ). Byun et al. ${ }^{18}$ clarify the discrepancy by indicating that although on gross inspection the eye appears to be closed, histological sections reveal that the eyelids have actually started to separate (Fig. 2C). This may explain why Doxanas and Anderson ${ }^{22}$ reported separation at 24 weeks. Sevel correctly points out that eyelid separation is a continuous process and not a short-term event, and even goes further to claim that several tissue strands normally persist between both eyelids until term. ${ }^{14}$ However, if this statement were accurate, clinicians would have reported isolated ankyloblepharon filiforme adnatum more often. Histologic sections reveal that the eyelids start to separate nasally and anteriorly then extend temporally and posteriorly by gradual sloughing of periderm cells into the amniotic fluid. ${ }^{13,14,18}$ The anterior segment of the globe also begins to mature during the same period. The corneal epithelium, which was continuous with the surface ectoderm prior to eyelid fusion and was 4 layers thick, undergoes an initial reduction to 2 layers. But by the time the eyelids separate, the cornea has almost fully matured developing 5 layers of epithelium. ${ }^{14}$

Until recently eyelid separation has lacked any reasonable explanation. It was mistakenly attributed to keratinization of the intermediate cell layer, which would presumably soften and help disengage the cells in the junctional region. ${ }^{11,13,14,24,34}$ Indeed, immunohistochemical studies have demonstrated that keratin-positive cells show significant changes during the process of eyelid separation, with over-expression of keratin 1 and
7 during the initial phase of eyelid separation, followed by the sudden and less potent expression of keratins 10, 13, 18, and 20 which occurs immediately before complete separation. ${ }^{39}$ These keratin-positive cells were observed on the epidermal surface and in the junctional region between both eyelids but not on the conjunctival surface. ${ }^{39}$ Other studies claimed that keratinization is only second in order of importance to holocrine production of lipids from the developing meibomian glands, ${ }^{10,13}$ and that the final stage of disjunction is attributed to traction by eyelid muscles. ${ }^{13,40}$ It is also possible - although not confirmed - that keratin expression inside the meibomian glands in the developing eyelid is a contributing factor to future eyelid separation. ${ }^{17,23}$ More recently, these old concepts have been partially challenged. ${ }^{18}$ Apoptosis has been identified as a possible key event (although not the only event) in eyelid separation. ${ }^{40}$ Interestingly, apoptosis is not only suggested to be responsible for eyelid separation but was demonstrated to play a role from the very beginning of eyelid fusion. It also appears to smooth out the proliferating periderm cells, eliminating any useless clumps and paving the way for a regular periderm sheet. ${ }^{40}$ Apoptosis-induced reduction in the size of the junctional area hypothetically facilitates differentiation and keratinization of the underlying epidermis, as it enables easy disruption of the junctional epithelial plug. It could be deduced that apoptosis plays a secondary but earlier role, and keratinization remains the ultimate and final process in eyelid separation. ${ }^{40}$

Finally, by week $24(232 \pm 18 \mathrm{~mm})$, after eyelid separation, the eyelids begin to take their nearly developed shape. ${ }^{14,18,22}$ At this stage, the orbicularis oculi muscle extends medially and laterally to be attached to the medial and lateral palpebral ligaments, respectively, ${ }^{14}$ the tarsal plate has lengthened and increased in width, the levator palpebrae superioris muscle, aponeurosis, and the orbital septum are all clearly visible, and 
the Müller's muscle is visibly attached to the superior border of the tarsus and is posterior to the levator aponeurosis. ${ }^{18}$ The thickened epimysium between the superior rectus and the levator palpebrae superioris finally starts to thin out. ${ }^{9,38}$ Fine fascial septae between both muscles, which are commonly encountered during ptosis surgery, may represent the remnants of this shared epimysium..$^{9,38}$ By week $28(267 \pm 27 \mathrm{~mm})$, the differentiation of the orbicularis oculi muscle is almost complete, and the pretarsal, preseptal, and orbital components are clearly discerned. ${ }^{10}$ Byun et al. ${ }^{18}$ worked out in detail meibomian gland morphogenesis and showed that by the 28th week, meibomian glands span half the length of the tarsal plate. Opening of the puncta onto the eyelid margin is also observed around the 28th week after the eyelids separate. ${ }^{30}$

By week $32(301 \pm 33 \mathrm{~mm})$, the upper eyelid central fat pad and the preseptal fat (which made their first appearance at weeks 14 and 18 , respectively) are well defined. ${ }^{18}$ Also by the $32 \mathrm{nd}$ week, meibomian glands increase in length and are present in two-thirds of the length of the tarsal plate (Fig. 2D). ${ }^{14}$ Initially, the meibomian glands of the upper and lower eyelids are roughly equal in length, and only later do the glands in the upper eyelid outpace their lower counterpart in vertical growth. ${ }^{21}$

The onset of dynamic eyelid movements (blinking) has rarely been scrutinized in detail in the literature. In 1985, Birnholz ${ }^{41}$ observed the earliest evidence of blinking at the age of 33 weeks $(310 \pm 33 \mathrm{~mm})$ after orange color stimulation. However, Petrikovsky et al. ${ }^{42}$ later documented an earlier onset of fetal blinking ( 31 weeks, $292 \pm 33 \mathrm{~mm}$ ) in $90 \%$ of examined fetuses by ultrasonography with a slow spontaneous blink rate of about 6 blinks over a 60 -minute period. This escalated to a rate of about 15 blinks/hour after vibroacoustic stimulation. The actual onset of blinking may be much earlier, but the eyelids may be too small for dynamic movements to be detected by ultrasound. ${ }^{42}$ For reference purposes, the resting baseline blink rate in adults is around 19 to 20 times per minute. ${ }^{43}$ This low blink rate during fetal life is probably due to intrauterine conditions (liquid vs. atmospheric medium), and not due to a lack of maturation, ${ }^{25}$ because a more recent study showed that premature infants spontaneously blink 6 times more frequently than did similarly aged intrauterine fetuses in Petrovsky's study. ${ }^{25,42}$

Finally, by week $36(336 \pm 32 \mathrm{~mm})$, the eyelid is almost fully formed with smooth eyelid margins. ${ }^{10}$ The tarsal glands now occupy almost the entire length of the tarsal plate, the orbicularis oculi is now located directly subcutaneously, and the levator muscle is connected to the Müller's muscle. ${ }^{18}$

\section{MOLECULAR BASIS OF EYELID DEVELOPMENT AND THE MURINE MODEL}

For 2 decades, the mouse has been the favorite model for the study of mammalian organogenesis, because it has been possible to modify the mouse germ line with the addition, replacement, modification, and even deletion of genes through homologous recombination of embryonic stem cells. Modification of the germ line and creation of mouse mutants with techniques, such as null mutations, microdeletions, point mutations, gene insertions, or chromosomal rearrangement, have dramatically deepened our understanding of eyelid embryonic development. A key to the development of tissues and organs during embryogenesis in multicellular organisms is the synchronized interaction of various cells that would make up the entire body. In the developing embryo, cell-to-cell contact is established in a paracrine (short distance), endocrine (long distance), or direct cell-to-cell (juxtacrine signaling) manner. This complex system of communication is regulated throughout the body by 11 major signaling pathways defined by the signal transducer or ligand involved. ${ }^{26}$

At the molecular level, regulation of eyelid development requires a bidirectional mesenchyme-periderm interaction, which has been extensively studied in the mouse and is surprisingly complex, requiring 7 of those 11 signaling pathways (WNT, Sonic hedgehog, transforming growth factor, fibroblast growth factor, epidermal growth factor, Jun N-terminal kinase, and notch pathways), not less than 22 genes, and 2 modes of communication (juxtacrine and paracrine)..$^{23,37,44-48}$ The process is complex, yet delicately controlled. Some of the cytokines and growth factors expressed by those 7 signaling pathways may be differentially or solely expressed in the leading edge cells or in the inner edge cells during eyelid fusion, in the mesenchyme, or in the cornea, and some may even be over-expressed in the upper eyelid more than the lower eyelid. ${ }^{23,49,50}$ It seems plausible to state that eyelid embryogenesis is "orchestrated by highly compartmentalized and spatially segregated developmental signals," ${ }^{48}$ but exactly how they interact in vivo is not yet fully understood. ${ }^{46}$

In general, the unique processes taking place during human eyelid morphogenesis share common features throughout the mammalian family tree, particularly in viviparous mammals, ${ }^{17,37}$ and in mice in particular this process has been studied in detail in more than 70 research articles in the past 25 years. Despite their similarities, embryologic mimicry between mouse and human eyelid is not absolute. Mouse eyelid formation, which starts at embryonic day 11 and fuses between embryonic days 15 and 16, does not start to reopen except at postnatal day 12 to 14 , and by postnatal day 16 eyelid separation is complete. This is in stark contrast to humans where the entire process is completed in utero. ${ }^{23,31,32,37,48}$ Understandably this in utero realization of eyelid fusion and separation is responsible for the scarcity of data in humans, which makes the murine model extremely helpful to identify eyelid developmental defects, particularly those associated with eyelid fusion and reopening failure..$^{15,18,48}$ Indeed, several genetic errors can lead to the well-studied defect in mice called eyelid open at birth phenotype. As of October 2015, the Mouse Genome Informatics lists 151 gene defects associated with this phenotype up from 138 in 2014 , and the number is likely to rise in the future with complete or partial knockout of new genes. ${ }^{47,48,51}$

\section{CLINICAL IMPLICATIONS}

Although a systematic appraisal of congenital eyelid anomalies is beyond the scope of the current review, several valid clinical points can be inferred from studying the process of eyelid morphogenesis, principally the Yin and Yang of eyelid fusion and separation. In mammals, eyelid fusion serves several important functions; it is crucial for differentiation of eyelid appendages, and also imparts a protective function on the developing components of the eye, especially the cornea. ${ }^{14,23,37}$ It is of crucial importance that the eyelids fuse before commencement of renal function $(70 \mathrm{~mm}$ stage), because as the kidneys start to function, the urea, uric acid, and creatinine content of amniotic fluid increases significantly. ${ }^{14}$ An additional surprising advantage of eyelid closure that was demonstrated recently is that it offers mechanical and structural support for formation of ocular adnexal structures as evidenced by experimental evidence of extraocular muscle defects, tarsal hypoplasia, and a blunted levator muscle, which could not reach beyond the superior fornix in 7 different mutant mouse strains all with an eyelid open at birth phenotype abnormality. ${ }^{48}$ Whether a transient or reversible eyelid fusion abnormality that goes undetected at birth could be implicated in some cases of congenital ptosis in humans through mechanical or genetic means is theorized but remains unproved and difficult to validate. ${ }^{48}$ 
The periderm cells, which are closely tied to the development of the eyelids, are also concomitantly involved in temporary fusion of the digits. ${ }^{37,52}$ This point is of significant clinical importance because patients with Fraser syndrome may suffer from syndactyly along with eyelid fusion anomalies. ${ }^{53}$ The fact that periderm cells are precursors for the development of the tarsal conjunctiva, coupled with the fact that corneal morphogenesis relies on some of the same bidirectional mesenchymeepithelium interactions that help shape the future eyelids, ${ }^{54}$ may explain why in cryptophthalmos, corneopalpebral adhesions are a significant cause of morbidity. ${ }^{55}$

It has been suggested recently that the process of adult wound healing, particularly skin healing that involves a synchronized series of tissue remodeling and tissue movements, is remarkably similar to the embryonic process of eyelid fusion. A process akin to "reawakening" of dormant morphogenetic tissue building mechanisms occurs when adult tissues are injured. ${ }^{35,49}$ Several transcription molecules involved in eyelid development also play a critical role in wound healing..$^{32,35,49}$ It would seem plausible to suggest that "repair recapitulates morphogenesis" with almost identical cytoskeletal machinery. ${ }^{33}$ One single remarkable exception is that eyelid morphogenesis usually results in a perfect outcome, whereas the final outcome in adult wound healing may involve an inflammatory response and result in scarring and tissue deformity. This could be an evolutionary response to protect adults against microbial invasion if the skin barrier is broken, whereas the sealed environment of the embryo does not require such protection against infection. ${ }^{50}$ The mechanism of re-epithelialization itself may be different, and several other factors may help explain this discrepancy. Embryonic eyelids fuse by first advancing filopdia and lamellipodia, which reach out and interdigitate with the opposite eyelid followed by closure of the gap in a sweeping pursestring like manner by forming a homogenous cable of contractile actin. In adults, on the other hand, the skin heals by filopodial and lamellipodial crawling of cells only without involvement of the actin cytoskeleton. ${ }^{35,50,56}$ Different collagens and keratins are involved, and signaling pathways are also differentially expressed in adult wound healing versus embryonic eyelid fusion, or even fetal wound healing, which also appears to be a relatively scarless process. ${ }^{56,57}$ Finally, while macrophages are actively involved in the adult wound healing process to clear cellular debris and release growth factors like TGF $\beta 1$, which appears to be instrumental in mediating a tissue-mediated fibrotic response, embryonic eyelid fusion seems to be a "macrophageless" process. ${ }^{35,56,57}$

In conclusion, the authors have summarized the recent developments in eyelid morphogenesis, a temporally precise and morphogenetically intricate process that requires the cells to fold, proliferate, migrate, fuse, and later separate in a synchronized and timely manner. However, several questions remain unanswered: how closely does the development of the eyeball, the eyelid, and the rest of the ocular adnexal structures exert an influence on each other; how do all those separate signaling pathways that orchestrate eyelid development synchronize and communicate with each other in utero; how can the reactivation of some of these pathways, instrumental as they are in embryonic life, be detrimental to adults by promoting the motility and invasiveness of malignant cells, ${ }^{58}$ and can they be selectively turned off or inactivated to slow the spread of malignancies; can the parallels between embryonic eyelid fusion and adult wound healing be exploited pharmaceutically to help fend off scarring in fully mature mammalian specimens; can the full identification of the signaling cascades and transcription molecules involved in eyelid fusion serve as a blueprint for developing potential molecular therapies for targeting congenital eyelid and ocular surface fusion abnormalities; and how do the unique phenomena occurring during eyelid development result in congenital ptosis.

\section{REFERENCES}

1. Gasser R. The virtual human embryo. Available at: http://virtualhumanembryo.lsuhsc.edu/. Accessed October 24, 2015.

2. Salomon LJ, Alfirevic Z, Bilardo CM, et al. ISUOG practice guidelines: performance of first-trimester fetal ultrasound scan. Ultrasound Obstet Gynecol 2013;41:102-13.

3. Schoenwolf G, Bleyl, S, Brauer P, Francis-West P. Origin of epidermis and dermis of skin. In: Schoenwolf G, Bleyl, S, Brauer P, FrancisWest P, eds. Larsen's Human Embryology. 4th ed. Philadelphia, PA: Churchill Livingstone Elsevier, 2009. Available at: https://www. inkling.com/read/larsens-human-embryology-schoenwolf-bleylbrauer-francis-west-4th/chapter-7/origin-of-epidermis-and-dermis. Accessed November 5, 2013.

4. Lee RT, Asharani PV, Carney TJ. Basal keratinocytes contribute to all strata of the adult zebrafish epidermis. PLoS One 2014;9:e84858.

5. Smyth I, Scambler P. The genetics of Fraser syndrome and the blebs mouse mutants. Hum Mol Genet 2005;14 Spec No. 2:R269-74.

6. Petrou P, Chiotaki R, Dalezios Y, et al. Overlapping and divergent localization of Frem 1 and Fras1 and its functional implications during mouse embryonic development. Exp Cell Res 2007;313:910-20.

7. Tao H, Shimizu M, Kusumoto R, et al. A dual role of FGF10 in proliferation and coordinated migration of epithelial leading edge cells during mouse eyelid development. Development 2005;132:3217-30

8. Hayashi Y, Liu CY, Jester JJ, et al. Excess biglycan causes eyelid malformation by perturbing muscle development and TGF-alpha signaling. Dev Biol 2005;277:222-34.

9. Dutton JJ. The eyelids and anterior orbit. In: Dutton JJ, ed. Atlas of Clinical and Surgical Orbital Anatomy. Philadelphia, PA: W.B. Saunders Company, 1994:113-38.

10. Barishak YR. Embryology of the eye and its adnexae. Dev Ophthalmol 1992;24:1-142.

11. Cook CS, Ozanics V, Jakobiec FA. Prenatal development of the eye and its adnexa. In: Tasman W, Jaeger EA, eds. Duane's Foundations of Clinical Ophthalmology. Philadelphia, PA: Lippincott, 1994: 1:2. Available at: http://www.oculist.net/downaton502/prof/ebook/ duanes/pages/v7/v7c002.html. Accessed June 13, 2014.

12. Hill M. Brief timeline of historical developments on the eye and its embryology. Available at: http://embryology.med.unsw.edu. au/embryology/index.php?title=2012_Group_Project_1\#cite_ref. Accessed December 14, 2013.

13. Andersen H, Ehlers N, Matthiessen ME. Histochemistry and development of the human eyelids. Acta Ophthalmol (Copenh) 1965;43:642-68.

14. Sevel D. A reappraisal of the development of the eyelids. Eye 1988;2:123-9.

15. Pearson AA. The development of the eyelids. Part I. External features. J Anat 1980;130(pt 1):33-42.

16. Nien CJ, Massei S, Lin G, et al. The development of meibomian glands in mice. Mol Vis 2010;16:1132-40.

17. Zhang H, Hara M, Seki K, et al. Eyelid fusion and epithelial differentiation at the ocular surface during mouse embryonic development. Jpn J Ophthalmol 2005;49:195-204.

18. Byun TH, Kim JT, Park HW, et al. Timetable for upper eyelid development in staged human embryos and fetuses. Anat Rec (Hoboken) 2011;294:789-96.

19. Corner GW, Smelser GK. The embryology of the eye-a motion picture. New York: Sturgis-Grants Productions. 1950 (cited from Byun et al. 2011).

20. Harris MJ, Juriloff DM. Eyelid development and fusion induced by cortisone treatment in mutant, lidgap-Miller, foetal mice. A scanning electron microscope study. J Embryol Exp Morphol 1986;91:1-18.

21. Whitnall SE. Eyelids. In: Whitnall SE, ed. The Anatomy of the Human Orbit and Accessory Organs of Vision. 1st ed. London: Henry Frowde and Hodder \& Stoughton, 1921:183-7.

22. Doxanos MT, Anderson RL. Embryology of the eyelids, lacrimal system and orbit. In: Doxanos MT, Anderson RL, eds. Clinical Orbital Anatomy. Vol. 1. Baltimore, MD: Williams and Wilkins, 1984:1-18.

23. Knop E, Knop N, Millar T, et al. The international workshop on meibomian gland dysfunction: report of the subcommittee on 
anatomy, physiology, and pathophysiology of the meibomian gland. Invest Ophthalmol Vis Sci 2011;52:1938-78.

24. Kapalanga J, Blecher SR. Histological studies on eyelid opening in normal male mice and hemizygotes for the mutant gene Tabby (Ta) with and without epidermal growth factor treatment. Exp Eye Res 1991;52:155-66.

25. Descroix E, Charavel M, Świątkowski W, et al. Spontaneous eyeblinking rate from pre-term to six-months. Cogent Psychology (serial online) 2015;2:1-14. Available at: http://www.tandfonline.com/ doi/full/10.1080/23311908.2015.1091062. Accessed November 15, 2015.

26. Perrimon N, Pitsouli C, Shilo BZ. Signaling mechanisms controlling cell fate and embryonic patterning. Cold Spring Harb Perspect Biol 2012;4:a005975.

27. Archie JG, Collins JS, Lebel RR. Quantitative standards for fetal and neonatal autopsy. Am J Clin Pathol 2006;126:256-65.

28. Saleh GM, Hussain B, Verity DH, et al. A surgical strategy for the correction of Fraser syndrome cryptophthalmos. Ophthalmology 2009;116:1707-1712.e1.

29. De la Cuadra-Blanco C, Peces-Pena MD, Janez-Escalada L, et al. Morphogenesis of the human excretory lacrimal system. J Anat 2006;209:127-35

30. Ali MJ, Kakizaki H. Embryology of the lacrimal drainage system. In: Ali MJ, ed. Principles and Practice of Lacrimal Surgery. New Delhi, India: Springer, 2015: 9-15.

31. Alazami AM, Shaheen R, Alzahrani F, et al. FREM1 mutations cause bifid nose, renal agenesis, and anorectal malformations syndrome. Am J Hum Genet 2009;85:414-8.

32. Yu Z, Bhandari A, Mannik J, et al. Grainyhead-like factor Get1/ Grhl3 regulates formation of the epidermal leading edge during eyelid closure. Dev Biol 2008;319:56-67.

33. Tao H, Ono K, Kurose H, et al. Exogenous FGF10 can rescue an eye-open at birth phenotype of Fgf10-null mice by activating activin and TGFalpha-EGFR signaling. Dev Growth Differ 2006;48:339-46.

34. Findlater GS, McDougall RD, Kaufman MH. Eyelid development, fusion and subsequent reopening in the mouse. $J$ Anat 1993;183(pt 1):121-9.

35. Martin P, Parkhurst SM. Parallels between tissue repair and embryo morphogenesis. Development 2004;131:3021-34.

36. Shirakata Y, Kimura R, Nanba D, et al. Heparin-binding EGF-like growth factor accelerates keratinocyte migration and skin wound healing. J Cell Sci 2005;118(pt 11):2363-70.

37. Ohuchi H. Wakayama symposium: epithelial-mesenchymal interactions in eyelid development. Ocul Surf 2012;10:212-6.

38. Sevel D. The origins and insertions of the extraocular muscles: development, histologic features, and clinical significance. Trans Am Ophthalmol Soc 1986;84:488-526.

39. Teraishi T, Yoshioka M. Electron-microscopic and immunohistochemical studies of eyelid reopening in the mouse. Anat Embryol (Berl) 2001;204:101-7.
40. Mohamed YH, Gong H, Amemiya T. Role of apoptosis in eyelid development. Exp Eye Res 2003;76:115-23.

41. Birnholz JC. Ultrasonic fetal ophthalmology. Early Hum Dev 1985;12:199-209.

42. Petrikovsky BM, Kaplan G, Holsten N. Eyelid movements in normal human fetuses. J Clin Ultrasound 2003;31:299-301.

43. Wambier SP, Ribeiro SF, Garcia DM, et al. Two-dimensional video analysis of the upper eyelid motion during spontaneous blinking. Ophthal Plast Reconstr Surg 2014;30:146-51.

44. Xia Y, Karin M. The control of cell motility and epithelial morphogenesis by Jun kinases. Trends Cell Biol 2004;14:94-101.

45. Holbro T, Hynes NE. ErbB receptors: directing key signaling networks throughout life. Annu Rev Pharmacol Toxicol 2004;44:195-217.

46. Jin C, Chen J, Meng Q, et al. Deciphering gene expression program of MAP3K1 in mouse eyelid morphogenesis. Dev Biol 2013;374:96-107.

47. Meng Q, Xia Y. c-Jun, at the crossroad of the signaling network. Protein Cell 2011;2:889-98

48. Meng Q, Mongan M, Carreira V, et al. Eyelid closure in embryogenesis is required for ocular adnexa development. Invest Ophthalmol Vis Sci 2014;55:7652-61.

49. Jin C, Yin F, Lin M, et al. GPR48 regulates epithelial cell proliferation and migration by activating EGFR during eyelid development. Invest Ophthalmol Vis Sci 2008;49:4245-53.

50. Hassemer EL, Endres B, Toonen JA, et al. ADAM17 transactivates EGFR signaling during embryonic eyelid closure. Invest Ophthalmol Vis Sci 2013;54:132-40.

51. Eyelids open at birth phenotype. Available at: http://www.informatics.jax.org/searches/Phat.cgi?id=MP:0001302. Accessed October $21,2015$.

52. Maconnachie E. A study of digit fusion in the mouse embryo. J Embryol Exp Morphol 1979;49:259-76.

53. van Haelst MM, Scambler PJ, Hennekam RC; Fraser Syndrome Collaboration Group. Fraser syndrome: a clinical study of 59 cases and evaluation of diagnostic criteria. Am J Med Genet A 2007;143A:3194-203.

54. Kao WW. Corneal morphogenesis during development and diseases. Eye Contact Lens 2010;36:265-8.

55. Tawfik HA, Abdulhafez MH, Fouad YA. Congenital upper eyelid coloboma: embryologic, nomenclatorial, nosologic, etiologic, pathogenetic, epidemiologic, clinical, and management perspectives. Ophthal Plast Reconstr Surg 2015;31:1-12.

56. Rolfe KJ, Grobbelaar AO. A review of fetal scarless healing. ISRN Dermatology(serial online) 2012;2012:698034. Available at:http://www.hindawi.com/journals/isrn/2012/698034/cta/. Accessed December 19, 2015.

57. Walmsley GG, Maan ZN, Wong VW, et al. Scarless wound healing: chasing the holy grail. Plast Reconstr Surg 2015;135:907-17.

58. Zenz R, Scheuch H, Martin P, et al. c-Jun regulates eyelid closure and skin tumor development through EGFR signaling. Dev Cell 2003;4:879-89. 